\title{
New Imaging Protocols for New Single Photon Emission CT Technologies
}

\author{
Piotr J. Slomka • Daniel S. Berman • Guido Germano
}

Published online: 20 April 2010

(C) The Author(s) 2010. This article is published with open access at Springerlink.com

\begin{abstract}
Nuclear cardiology practitioners have several new technologies available with which to perform myocardial perfusion single photon emission CT (MPS). These include dedicated small-footprint cardiac scanners, new stationary or semi-stationary three-dimensional detectors, and advanced software algorithms for optimal image reconstruction. These new technologies have been employed to reduce imaging time and radiation exposure. They require less technologist and camera time and offer improved patient comfort. They have potential for the overall cost reduction of MPS and at the same time for improved accuracy by increased resolution, or accurate attenuation correction. Furthermore, these new technologies offer potential for new protocols such as simultaneous dual isotope, new combinations of isotopes, stress only MPS, or dynamic first-pass imaging. In addition, new imaging technologies in coronary $\mathrm{CT}$ angiography (CCTA) allow novel hybrid stress only MPS/CCTA protocols with reduced radiation burden. Additional developments further improving efficiency and diagnostic accuracy of MPS are on the horizon.
\end{abstract}

Keywords Myocardial perfusion SPECT - Solid state detectors · Stress-only protocol $\cdot$ Hybrid imaging $\cdot$ Cardiac fusion - Simultaneous dual isotope - Fast cardiac SPECT. Dynamic imaging

\section{Introduction}

Myocardial perfusion single photon emission CT (MPS) is a widely used clinical test, with approximately 9 million

P. J. Slomka $(\bowtie) \cdot$ D. S. Berman · G. Germano

8700 Beverly Boulevard, Suite A047,

Los Angeles, CA 90048, USA

e-mail: SlomkaP@cshs.org studies being performed annually in the United States [1]. Until recently, typical scans were long procedures, requiring 15 to $20 \mathrm{~min}$ for each stress and rest MPS acquisition, to allow adequate imaging statistics. Additional consequences of long imaging times were frequent motion artifacts caused by patient motion during the scan and compromised patient comfort. Recent advances in new detector designs allow dramatic reduction of imaging times to less than $2 \mathrm{~min}$ in some cases, or alternatively, dramatic reductions in the radiation dose to the patient. Cameras now available from many vendors have increased photon sensitivity achieved by means of custom detector geometry and innovative designs of collimation (including multiple pinhole or slit hole collimators), focusing of the field of view on the heart and using solidstate detectors. The optimized geometry for cardiac imaging allows significantly reducing or totally eliminating the motion of the detector, with simultaneous collections of counts from the whole heart. The new cameras have often reduced footprint and allow easier patient positioning, and in many cases upright imaging. Faster imaging and more comfortable patient position results in reduced patient motion during acquisition. Additionally, three-dimensional (3D) character of the acquisition reduces the effect of patient motion on image quality.

These new camera designs are coupled with novel methods of image reconstruction. Improvements in reconstruction algorithms allow accurate resolution recovery and corrections of physical effects via iterative reconstruction algorithms. These new methods of image reconstruction can be implemented on standard cameras. Combination of the new hardware and the new reconstruction models allows simultaneous improvements in photon sensitivity and image resolution. Furthermore, list mode acquisition capabilities are now available, allowing arbitrary time reformatting of the data after the scan. Consequently, firstpass 3D dynamic imaging and kinetic modeling with 
stationary or semi-stationary single photon emission CT (SPECT) detectors is now possible, similar to what has been available in positron emission tomography (PET). Additionally, increased photon sensitivity can be utilized to pursue novel imaging protocols such as simultaneous dual isotope, or new combinations of isotopes allowing rapid stress/rest assessment. Recently we reviewed new hardware and software developments from a technical perspective [2•]. In this review article, we summarize current clinical applications and trials with emphasis on novel imaging protocols that are possible with the new generation of devices, including hybrid imaging protocols.

\section{New Cardiac SPECT Technologies}

Several vendors have developed hardware and software technologies allowing reduced imaging or dose time, improved patient comfort, and improved image quality. Digirad Inc. (Poway, CA) has developed a cardiac Cardius XPO camera, which is coupled with new reconstruction technology (n-speed). For imaging, the patient sits on a chair with his or her arms placed on an armrest above the detectors [3]. Digirad has also incorporated a low-cost approach to obtain $\mathrm{x}$-ray transmission scans for attenuation correction that can be implemented with the XPO camera. Another dedicated cardiac camera was developed by CardiArc (Canton, MI), optimized specifically for cardiac imaging and upright patient position [4]. Spectrum Dynamics (Haifa, Israel) has manufactured a solid-state dedicated cardiac system called D-SPECT with semi-stationary detectors, high-sensitivity collimation, and reconstruction optimized specifically for cardiac imaging. The patient is imaged in a semi-upright position with the left arm placed on top of the camera or in the supine position. General Electric Healthcare has developed a stationary imaging camera based on the multipinhole design, an alternative technique for photon collimation [5-7], and an array of cadmium zinc telluride pixilated detectors [8]. Patients are imaged in a supine position with their arms placed over their heads. Two examples of studies obtained with D-SPECT and GE Discovery NM 530c with comparable acquisition times and doses are shown in Fig. 1. Siemens has combined an astigmatic collimator, an optimized organ-of-interest centered acquisition, and iterative reconstruction in their IQ•SPECT system with CT-based attenuation correction (on Symbia platform) and energy window-based scatter correction [9]. This system allows hybrid MPS-CT imaging.

In parallel, efforts have been undertaken to improve image contrast and reduce noise levels by reconstruction algorithms that incorporate physical modeling of the detectors based on ordered subsets expectation maximization (OSEM) [10] and applied to data acquired on standard scanners. Philips (San Jose, CA) has developed a fast SPECT reconstruction algorithm (Astonish) that includes corrections for photon scatter, photon attenuation, and variations in spatial resolution and allows reduced imaging time. UltraSpect Inc. (Haifa, Israel) has developed a standalone workstation that utilizes the patented Wide Beam Reconstruction (WBR) algorithm [11, 12].

\section{Fast Imaging and Dose Reduction in Clinical Studies}

In the past 2 years, these new hardware systems and reconstruction techniques have been utilized clinically to reduce patient imaging time and radiation dose. Typically, these studies have been validated by comparing to studies performed with the same injected dose during the same patient visit on a conventional dual head SPECT camera. Data were analyzed by blinded visual or by quantitative analysis. Several of these studies combined data from multiple centers. Herzog et al. [13] performed phantom and clinical studies to establish the most optimal imaging time for the solid-state multi-pinhole Discovery NM 530c detectors and found that scan as short as 2 min with $327 \mathrm{MBq}$ mean dose and 3-minute rest scan with $915 \mathrm{MBq}$ provide equivalent images to 15 -minute acquisitions with the standard camera. A similar protocol was followed in 75 patients by the same group, and excellent correlation for per-vessel and per-patient perfusion defects was found with conventional SPECT [14]. In the United States, the Discovery NM 530c system was evaluated by the Emory University group [15•]. Similarly high correlations with conventional SPECT were found for perfusion defects and for left ventricular ejection fractions (LVEFs).

The D-SPECT camera was compared with a standard dual detector in 44 patients and reported shorter imaging times and higher MPS quality for the new camera, with high correlations in visual perfusion scores [16]. In another study, a novel stress Tl-201/rest Technetium-99m protocol, with total stress/rest imaging time reduced to less than $20 \mathrm{~min}$, including additional supine views, was described and compared to a standard $\mathrm{Tc} / \mathrm{Tc}$ protocol on the same fast camera (Fig. 2) [17•]. They concluded that $\mathrm{Tl} / \mathrm{Tc}$ protocol offers similar image quality and dosimetry $(11.9 \mathrm{mSv}$ vs $11.2 \mathrm{mSv} ; P=$ not significant), but results in much shorter total imaging time and offers potential for better depiction of stress lesions with physiologically superior Tl-201 agent (Fig. 3). Furthermore, this study demonstrated possibility of dual-position imaging for these new cameras with patient imaged in upright and supine positions. Two-position imaging allows improved diagnostic accuracy by resolving of subtle attenuation artifacts or patient motion as demonstrated in prone-supine conventional SPECT imaging [18]. In another large study of D-SPECT data from four US 
Fig. 1 Examples of two patient studies obtained with Discovery NM 530c (system 1; images courtesy of Dr. Ernest Garcia) and D-SPECT (system 2; images courtesy of Dr. Jack Ziffer), showing good-quality studies with comparable acquisition protocols (2-minute stress, 4-minute rest)

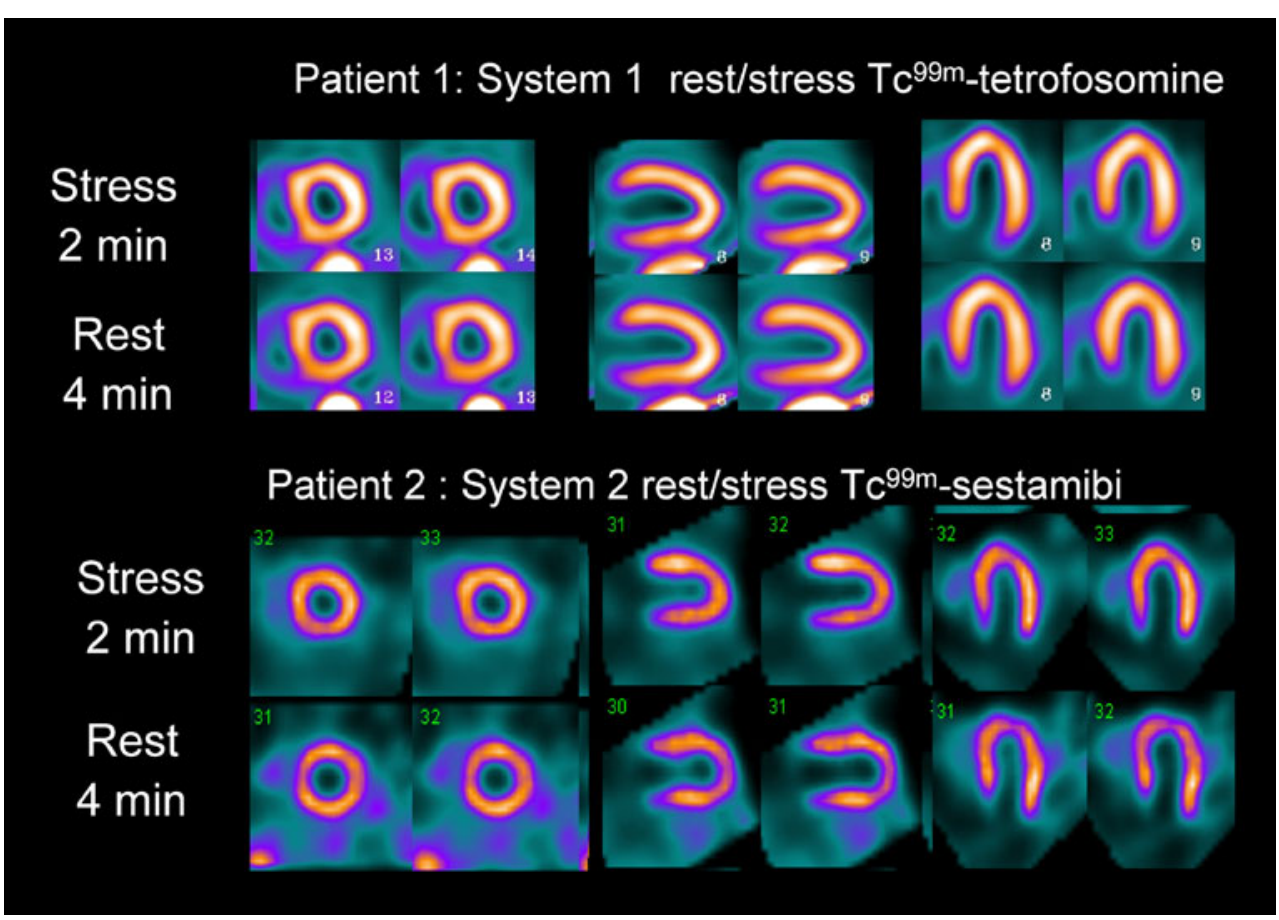

centers, Sharir et al. [19••] used fully automated quantitative software with normal limits developed for the new camera for their data analysis. They demonstrated that the quantitative total perfusion deficit [20] obtained with the fast protocol correlated linearly with stress and rest conventional SPECT ( $r=0.95$ and $r=0.97$, respectively; $P<0.0001$ ), and that the average amount of ischemia was higher in the highlikelihood patients, as compared to conventional SPECT. Similarly, LVEFs and LV volumes obtained with fully quantitative analysis [21] were highly correlated.

Fast imaging protocol for the Digirad cardiac system (majority with dual or triple head configuration) with optimized image reconstruction has been evaluated in a multicenter trial (11 centers) by Maddahi et al. [22]. They found that images from fast protocols were diagnostically equivalent in 444/448 (99\%) of the patients as determined by blinded visual analysis. Functional parameters ejection fraction, diastolic, and systolic volume were also highly correlated. With a conventional camera, fast imaging by means of optimized WBR reconstruction with image resolution recovery and novel approach to noise control was also evaluated [12]. This reconstruction was applied to data obtained on a conventional dual-head system (GE, Ventri) with high-resolution parallel-hole camera. DePuey et al. [23] reported equivalent summed stress and rest perfusion scores with similar image quality at rest and superior at stress for the in $1 / 4$ time WBR imaging as compared to full-time standard OSEM reconstruction. Similar to the other studies, functional parameters were also highly correlated.
Fig. 2 Fast T1-201

stress/Tc-99m rest protocol for adenosine (top) and exercise (bottom) myocardial perfusion SPECT protocol. At peak stress, patients were injected with T1-201. After a 5-minute post-stress monitoring period, supine and semi-upright (sitting) acquisitions were performed sequentially. Subsequently, Tc-99m sestamibi (mibi) or tetrofosmin (tetro) was injected, and a single 4-minute sitting rest acquisition was performed. (Modified from Berman et al. $[17 \bullet])$

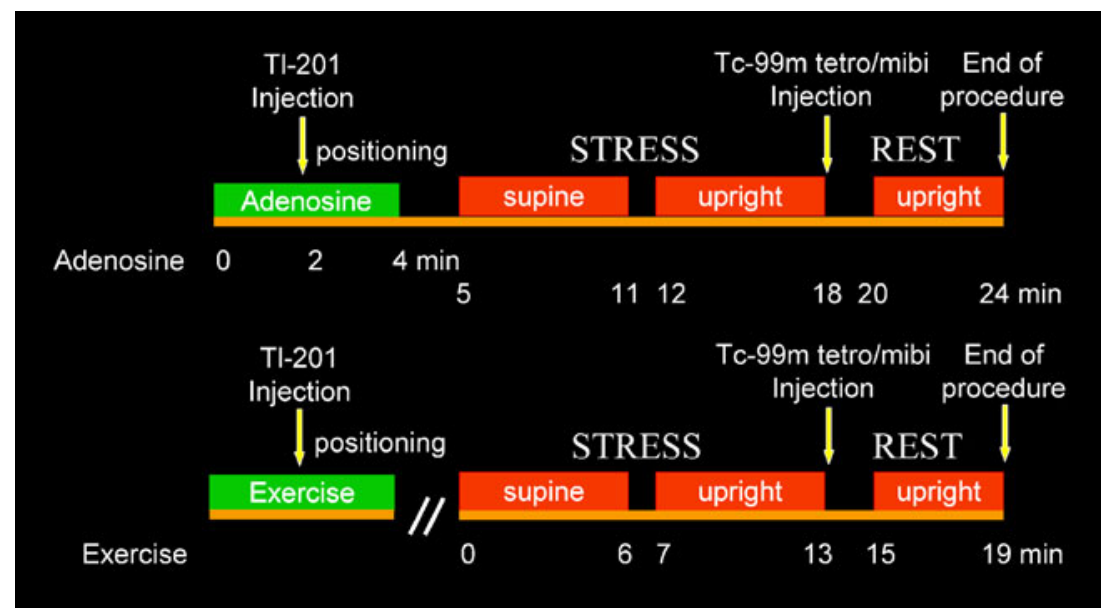




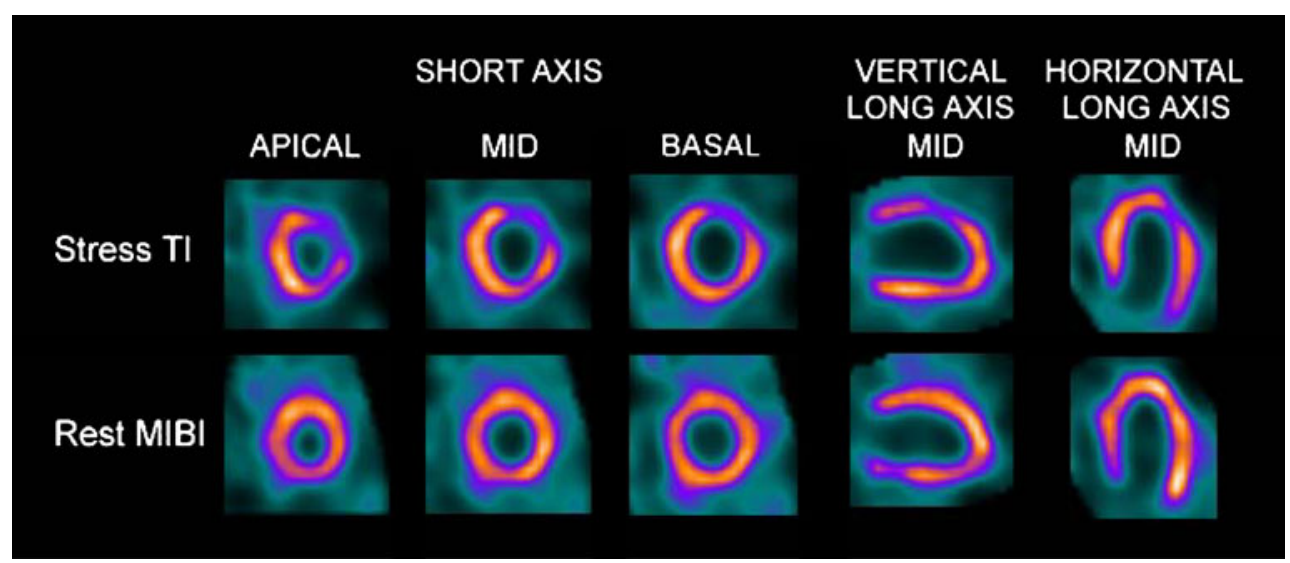

Fig. 3 Images of a 53-year-old man with no known coronary artery disease and hypertension, diabetes, hypercholesterolemia, and shortness of breath, acquired with fast ${ }^{201} \mathrm{~T} 1$ exercise stress $/{ }^{99 \mathrm{~m}} \mathrm{Tc}$ rest protocol showing large severe reversible defects in the anterior and lateral walls.

In other clinical reports, some preliminary results have been presented for the SPECT•IQ system, with imaging times as short as $4 \mathrm{~min}$. The Symbia T series systems also allow for obtaining of CT calcium scan in as little as $30 \mathrm{~s}$ during the same imaging session [24]. Preliminary study of fast 3-minute gated imaging with multi-pinhole system on conventional detectors has been also been presented by Koss et al. [25], which facilitates the study of segmental LV perfusion and contractile performance during infused dobutamine stress and during the recovery period following stress.

\section{Stress-Only MPS}

The effective imaging time and patient dose reduction could also be accomplished by means of eliminating the resting MPS scan. Stress-only MPS protocols with attenuation correction (AC) have been advocated in the past [26]. The stress-only approach increases laboratory efficiency, decreases cost, and improves patient convenience, while reducing radiation dose [27]. The Philips Astonish approach to reducing scan time allows simultaneous AC and MPS acquisition, reducing potential for any misregistration artifacts associated with $\mathrm{CT}$ AC. It has been demonstrated that half-time acquisitions, when compared to standard (filtered back-projection) FBP processing, have equivalent diagnostic accuracy with both Astonish [28] and GE Evolution systems [29]. Bateman et al. [30•] conducted a study to determine the clinical feasibility of stress-only protocol with reduced scan time and simultaneous acquisition of $\mathrm{AC}$ scan. They found that simulated half-time scans (obtained by reconstruction sampling from full-time scans) when compared to standard FBP reconstruction without AC had similar quality and
Coronary angiography revealed $70 \%$ stenosis in the mid left anterior descending artery and the second diagonal branch. Percutaneous coronary intervention and stent implantation were subsequently performed in these two vessels

interpretive certainty, with equivalent summed perfusion scores obtained by blinded visual analysis. Importantly, in this study 92 of 118 cases had available correlating angiography and the diagnostic performance for the stress-only halftime AC MPS imaging was equivalent to standard full-time FBP studies. Therefore, the authors concluded that the stressonly AC MPS approach with optimized reconstruction has the potential to significantly improve MPS efficiency without reducing test accuracy. Additionally, stress-only scan reduces the patient radiation dose to less than $8 \mathrm{mSv}$, which could be further reduced by using standard rather than half-time acquisition times.

Recent peer-reviewed clinical studies referenced above, which utilize the new technology in order to reduce imaging time, are summarized in Table 1.

\section{Novel MPS Protocols}

Beyond simply reducing the acquisition time or patient radiation dose, there are several reports of novel MPS protocols, which take advantage of superior imaging characteristics of new devices or availability of new modalities for hybrid imaging. These include techniques for simultaneous dual isotope imaging, dynamic imaging, and hybrid coronary CT angiography (CCTA)/MPS imaging.

\section{Simultaneous Dual Isotope}

Routine simultaneous dual isotope would reduce the imaging time in half compared to the separate stress and rest study. The superior energy resolution of the new detectors can allow better separation of photons from different isotopes, which can result in much improved cross-talk correction [32]. In addition, stationary detector 
Table 1 Recent clinical studies demonstrating possibility of shortened imaging times with MPS

\begin{tabular}{|c|c|c|c|c|c|}
\hline Study & System & $N$ & Protocol & Injected dose & Acquisition time \\
\hline Herzog et al. [13] & Discovery NM 530c & 20 & $\begin{array}{l}\text { 1-day }{ }^{99 \mathrm{~m}} \mathrm{Tc} \text {-tetrofosmin } \\
\text { adenosine stress-rest }\end{array}$ & Stress/rest $8.8 / 25.1 \mathrm{mCi}$ & $\begin{array}{l}3 \mathrm{~min} / 2 \mathrm{~min} \\
\text { optimal }\end{array}$ \\
\hline Buechel et al. [14] & Discovery NM 530c & 75 & $\begin{array}{l}\text { 1-day }{ }^{99 \mathrm{~m}} \mathrm{Tc} \text {-tetrofosmin } \\
\text { adenosine stress-rest }\end{array}$ & Stress/rest 9.1/25.6 mCi & $3 \mathrm{~min} / 2 \mathrm{~min}$ \\
\hline Maddahi et al. [22] & Digirad 2/3-head & $\begin{array}{l}189 \text { 3-head } \\
239 \text { 2-head }\end{array}$ & Rest/stress ${ }^{99 \mathrm{~m}} \mathrm{Tc}$ & Rest/Stress $7-10 \mathrm{mCi} / 20-30 \mathrm{mCi}$ & $\begin{array}{l}4.8 \mathrm{~min} / 4.3 \mathrm{~min} \\
6.5 \mathrm{~min} / 4.3 \mathrm{~min}\end{array}$ \\
\hline Esteves et al. [15•] & Discovery NM $530 \mathrm{c}$ & 168 & $\begin{array}{l}\text { 1-day }{ }^{99 \mathrm{~m}} \mathrm{Tc} \text {-tetrofosmin } \\
\text { rest/stress }\end{array}$ & Rest/stress $10-15 / 30-45 \mathrm{mCi}$ & $4 \mathrm{~min} / 2 \mathrm{~min}$ \\
\hline Sharir et al. [16] & D-SPECT & 44 & $\begin{array}{l}\text { 1-day }{ }^{99 \mathrm{~m}} \mathrm{Tc} \text { stress/rest } \\
\text { sestamibi }\end{array}$ & Stress/rest 11/28 mCi & $4 \mathrm{~min} / 2 \mathrm{~min}$ \\
\hline Berman et al. [17•] & D-SPECT & $\begin{array}{l}374 \\
262\end{array}$ & $\begin{array}{l}\text { Stress } \mathrm{Tl} / \mathrm{rest}{ }^{99 \mathrm{~m}} \mathrm{Tc} \\
\text { Rest/stress Tc/Tc }\end{array}$ & $\begin{array}{l}\text { Stress/rest } \mathrm{Tl} / \mathrm{Tc} 2-2.5 / 8-10 \mathrm{mCi} \\
\mathrm{Tc} / \mathrm{Tc} 8-10 / 25-40 \mathrm{mCi}\end{array}$ & $\begin{array}{l}6 \mathrm{~min} / 4 \mathrm{~min} \\
4 \mathrm{~min} / 2 \mathrm{~min}\end{array}$ \\
\hline Sharir et al. [19••] & D-SPECT & 238 & $\begin{array}{l}\text { Rest/stress tetrofosmin/ } \\
\text { sestamibi }{ }^{99 \mathrm{~m}} \mathrm{Tc}\end{array}$ & Rest/stress $10 / 30 \mathrm{mCi}$ & $4 \mathrm{~min} / 2 \mathrm{~min}$ \\
\hline Gambhir et al. [31] & D-SPECT & 18 & $\begin{array}{l}\text { 1-day }{ }^{99 \mathrm{~m}} \mathrm{Tc} \text {-sestamibi } \\
\text { stress/rest }\end{array}$ & Stress/rest 11/28 mCi & $4 \mathrm{~min} / 2 \mathrm{~min}$ \\
\hline DePuey et al. [23] & $\begin{array}{l}\text { UltraSPECT Widebeam } \\
\text { Reconstruction }\end{array}$ & 209 & Rest/stress ${ }^{99 \mathrm{~m}} \mathrm{Tc}$-sestamibi & Rest/stress $9 / 32 \mathrm{mCi}$ & $4.5 \mathrm{~min} / 4 \mathrm{~min}$ \\
\hline Bateman et al. [30•] & Stress only Astonish & 110 & Stress only ${ }^{99 m} \mathrm{Tc}$-sestamibi & Stress $25-35 \mathrm{mCi}$ & $6 \mathrm{~min} /-$ \\
\hline
\end{tabular}

MPS myocardial perfusion SPECT, SPECT single photon emission CT

system design eliminates the projection consistencies that would further confound the simultaneous dual isotope imaging. The improved statistical content of new scanners is also a key factor in supporting the ability to image multiple isotopes simultaneously. A natural application is to simultaneously image ${ }^{99 \mathrm{~m}} \mathrm{Tc}$ and ${ }^{201} \mathrm{Tl}$ in a combined rest/ stress protocol as originally proposed for standard cameras [33], but never implemented clinically due to technical difficulties.

A proof-of-concept of simultaneous dual-isotope imaging on a multi-pinhole system was recently demonstrated by Steele et al. [34], who used $222 \mathrm{MBq}$ of ${ }^{99 \mathrm{~m}} \mathrm{Tc}$ injected at rest and $148 \mathrm{MBq}$ of ${ }^{201} \mathrm{Tl}$ injected $1 \mathrm{~min}$ before the end of exercise and performed simultaneous dual-isotope imaging of ${ }^{201} \mathrm{Tl}$ (stress) and ${ }^{99 \mathrm{~m}} \mathrm{Tc}$ (rest) immediately after completion of exercise, by the three-detector 18-pinhole system, during a single 20-minute imaging session. Ben-Haim et al. [35] reported the feasibility of simultaneous dual-isotope application with the solid-state D-SPECT camera. They studied 24 patients with simultaneous dual-isotope imaging with $80 \mathrm{MBq}$ of ${ }^{201} \mathrm{Tl}$ injected at rest and $250 \mathrm{MBq}$ of ${ }^{99 \mathrm{~m}} \mathrm{Tc}$ sestamibi injected during adenosine infusion. Images were collected in a 15-minute list mode simultaneous dual radionuclide (rest/stress) gated acquisition with a spillover and scatter correction method, specifically designed for solid-state camera. Comparable diagnostic performance and image quality to conventional SPECT with a separate rest ${ }^{201} \mathrm{Tl}$ acquisition on D-SPECT has been demonstrated. The proposed imaging protocol for this application is shown in Fig. 4.

\section{Dynamic Imaging}

The potential for absolute measurement of flow and flow reserve has remained the key advantage of PET over SPECT [36]. The new SPECT camera designs, however, with stationary detectors such as the multi-pinhole systems $[8$, 37], or systems with semi-stationary detectors such as DSPECT design, where the whole $3 \mathrm{D}$ view is captured with limited detector motion in a few seconds, may allow first-pass MPS imaging. The proof-of-concept of such studies for the Discovery NM 530c platform has been demonstrated by Huang et al. [38] with the NCAT digital phantom simulations and one-compartment modeling, for ${ }^{99 \mathrm{~m}} \mathrm{Tc}$-teboroxime. They concluded that this stationary MPS system can provide a parametric image of myocardial blood flow for this myocardial perfusion agent. A group at Brigham and Women's Hospital in Boston, MA, in collaboration with our center, is currently exploring the quantification of regional and global estimates of coronary flow reserve on the D-SPECT system with ${ }^{99 \mathrm{~m}} \mathrm{Tc}$ sestamibi [39]. MPS imaging is performed with 10 and $30 \mathrm{mCi}$ of ${ }^{99 \mathrm{~m}} \mathrm{Tc}$ sestamibi injected at rest and during vasodilator stress, respectively, with imaging starting immediately with the intravenous bolus injection of radiotracer. Using list mode capabilities of D-SPECT device, the 4-minute stress and rest image data are reconstructed into 703.5 -second frames. Estimates of the myocardial perfusion reserve can be derived with one-compartment model implemented within the Cedars-Sinai kinetic modeling QPET software in a fully automated mode [40]. Estimates of myocardial reserve can be obtained on a per-vessel or per- 
Fig. 4 Illustration of the simultaneous dual isotope (SDI) protocol for the solid state camera

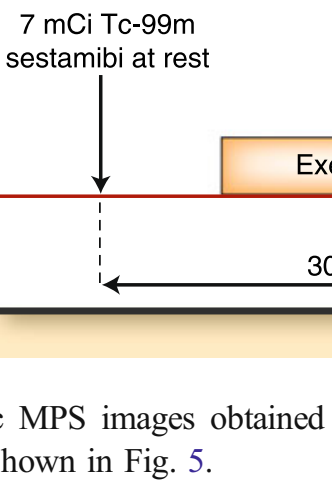

segment basis. Example of dynamic MPS images obtained with this novel imaging protocol is shown in Fig. 5.

\section{Hybrid Imaging}

A novel concept in modifying the imaging protocols is to employ hybrid technology [41] that may include novel software tools for image fusion. Specifically, recent advances in CCTA allow routine dose reduction in prospective mode to the $2 \mathrm{mSv}$ range [42]. CCTA has been shown to have a very high negative predictive value in detection of coronary artery disease. These new developments allow a combined CCTA-MPS imaging protocol with acceptable radiation burden. Exploiting this concept,

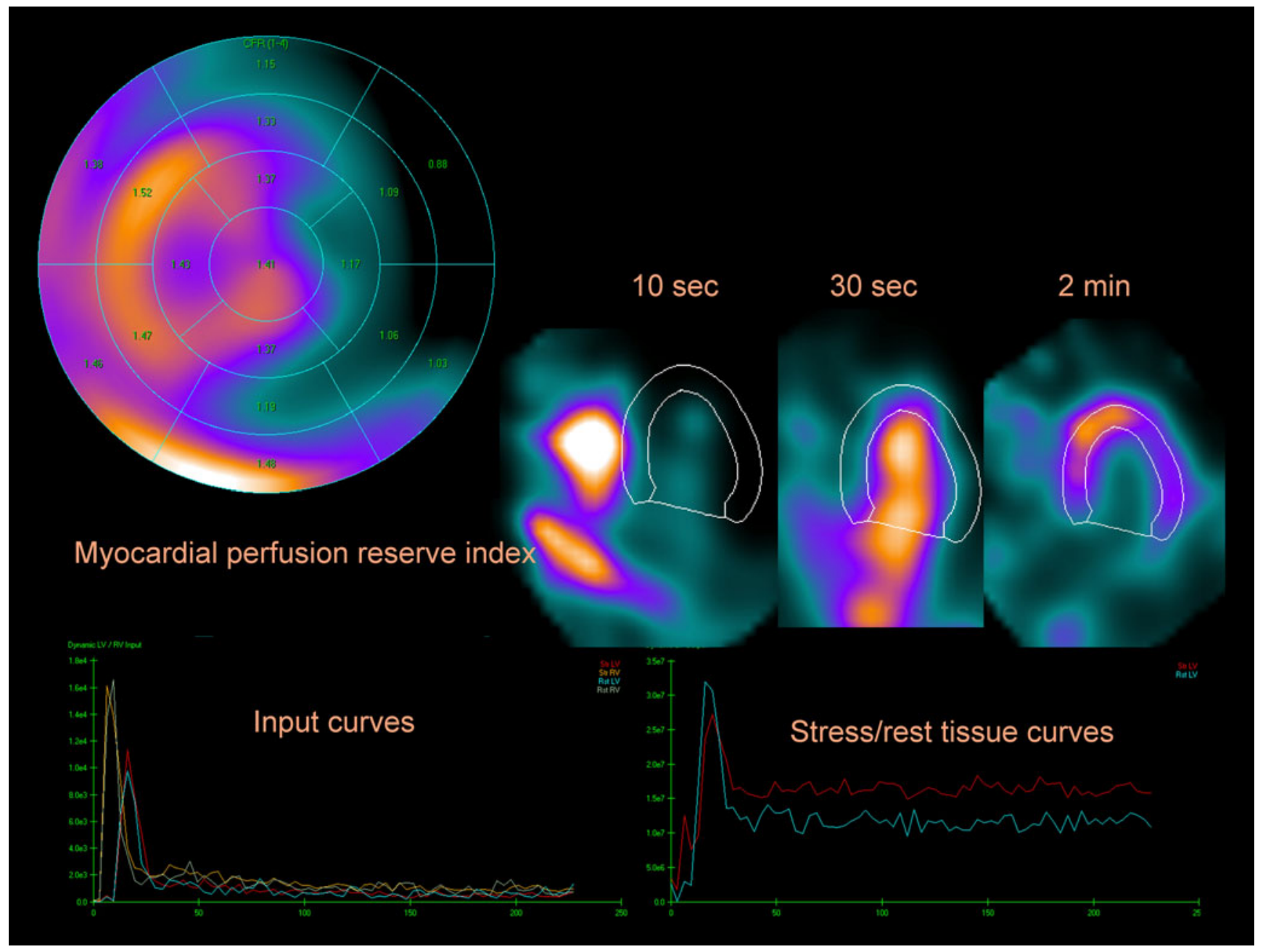

Fig. 5 Dynamic quantitative analysis of abnormal study obtained on D-SPECT system showing segmental analysis of myocardial perfusion index with segmental values (top left), dynamic images, myocardial perfusion SPECT images (top left), input curves (bottom left), and output stress and rest curves (bottom right). (Data courtesy of Marcelo Di Carli, Brigham \& Women's Hospital) 
Husmann et al. [43•] demonstrated the feasibility of stressonly MPS/CCTA protocol. In the study, 1-day adenosine stress/rest MPS using a dose of 300 and $900 \mathrm{MBq}$ of ${ }^{99 \mathrm{~m}} \mathrm{Tc}-$ tetrofosmin, respectively, for stress and rest, with low-dose CT attenuation correction, was combined with CCTA obtained on a separate GE VCT 64-slice scanner in 100 patients. The total patient time scheduled on the hybrid CCTA/stress-MPS examination protocol was $130 \mathrm{~min}$, which is shorter than total times during current 1-day stress/rest MPS protocols. It should be noted that this protocol could be significantly shortened if rest scans were not performed. All CCTA examinations in this study were performed in the $90 \mathrm{~min}$ between the first injection of

${ }^{99 \mathrm{~m}}$ Tc-tetrofosmin and stress data acquisition. They have shown that by using stress-only and prospectively gated CCTA data the combined dose would be $5.4 \pm 0.8 \mathrm{mSv}$, and such hybrid combination offers additional diagnostic information as compared to stress/rest study, without increased radiation exposure [43•]. Furthermore, hybrid cardiac imaging offered a high confidence in image interpretation, as equivocal findings in either of the scans can be resolved by the complementary modality. The principal drawback of this approach is that clinicians usually do not know ahead of time which patients will require both CCTA and MPS studies, such that sequential application of the methods, only when needed, may prove to be the more cost effective approach.

\section{Future Directions}

To summarize, many novel protocols have been described recently taking advantage of new detector design and new algorithms for software reconstruction. Primarily, the new clinical studies have aimed to reduce the total imaging time and scanning efficiency. However, in view of recent increased awareness of potential radiation exposure risks, it is possible that these protocols will be modified to reduce the radiation dose instead. It is conceivable that this can be customized based on the patient age, for example [44], since the radiation risk models assume that risk is inversely proportional to patient age. Similarly, to date, stress-only protocols have been demonstrated with conventional patient dosing. It is feasible that an aggressive dose-reducing protocol could involve fulltime stress-only scan with reduced dose. Similarly, the hybrid CCTA/stress MPS protocol could be performed with the use of the new efficient detectors, allowing a further dose reduction. The CCTA technology is also advancing rapidly, and recently excellent image quality was reported for new high-pitch spiral CCTA at a consistent dose below $1.0 \mathrm{mSv}$ [45]. In addition, it should be possible to selectively eliminate the stress MPS scan, for example if CCTA was normal [41], reducing the effective radiation burden and procedure cost. Additional improvements in hybrid imaging diagnostic accuracy could be accomplished by accurate image fusion aiding visual [46] or quantitative [47] analysis.

As the new efficient MPS hardware and software designs are being evaluated clinically, there are preclinical efforts ongoing that demonstrate that even higher sensitivity and efficiency can be achieved in cardiac SPECT. For example, Zeng and Stevens [48] theoretically demonstrated that multidivergent-beam collimators could be used for stationary cardiac SPECT and that this design has the potential to be superior to currently available multi-pinhole systems in terms of sensitivity by a factor of 2 , maintaining the same image resolution. In another report, theoretical calculations for multi-pinhole designs with curved detectors fitted to pinholes demonstrated potential of close to $50 \%$ improvement [49] as compared to the latest multi-pinhole designs clinically available.

\section{Conclusions}

Nuclear cardiology equipment and associated software have undergone very significant advances in the recent years. These new developments have been employed to test new imaging protocols with improved patient comfort, reduced imaging time, and reduced radiation dose. Novel protocols such as simultaneous dual-isotope, stress-only acquisitions, dynamic imaging, and hybrid multimodality protocols are being explored to further reduce imaging time and also improve the diagnostic information. Additional improvements in efficiency of cardiac SPECT technologies are possible, allowing further reductions in imaging time and radiation dose.

Disclosure Dr. Berman has equity position in Spectrum Dynamics Inc., and all of the authors participate in software royalties from Cedars-Sinai Medical Center.

Open Access This article is distributed under the terms of the Creative Commons Attribution Noncommercial License which permits any noncommercial use, distribution, and reproduction in any medium, provided the original author(s) and source are credited.

\section{References}

Papers of particular interest, published recently, have been highlighted as:

- Of importance

•- Of major importance

1. Dilsizian V, Narula J: Atlas of Nuclear Cardiology, edn 3. Springer; Philadelphia, PA: 2009.

2. - Slomka PJ, Patton JA, Berman DS, Germano G: Advances in technical aspects of myocardial perfusion SPECT imaging. J Nucl Cardiol 2009, 16:255-276. This is a comprehensive review of the technical aspects of the new systems and software. 
3. Lewin HC, Hyun MC: A clinical comparison of an upright triplehead digital detector system to a standard supine dual-head gamma camera [abstract]. J Nucl Cardiol 2005, 12:113.

4. www.CardiArc.com. Accessed May 30, 2008.

5. Jaszczak RJ, Li J, Wang H, et al.: Pinhole collimation for ultrahigh-resolution, small-field-of-view SPECT. Phys Med Biol 1994, 39:425-437.

6. Schramm NU, Ebel G, Engeland U, et al.: High-resolution SPECT using multipinhole collimation. IEEE Transactions on Nuclear Science 2003, 50:315-320.

7. Beekman FJ, Vastenhouw B: Design and simulation of a highresolution stationary SPECT system for small animals. Phys Med Biol 2004, 49:4579-4592.

8. Volokh L, Hugg J, Blevis I, et al.: Effect of detector energy response on image quality of myocardial perfusion SPECT. Paper presented at IEEE Nuclear Science Symposium and Medical Imaging Conference. Dresden, Germany; October 19-26, 2008.

9. Vija H, Chapman J, Ray M: IQ•SPECT technology White Paper. Siemens Medical Solutions USA Molecular Imaging; 2008:1-7.

10. Hudson HM, Larkin RS: Accelerated image reconstruction using ordered subsets of projection data. IEEE Transactions on Medical Imaging 1994, 13:601-609.

11. Ultraspect. www.UltraSPECT.com. Accessed September 6, 2008.

12. Borges-Neto S, Pagnanelli RA, Shaw LK, et al.: Clinical results of a novel wide beam reconstruction method for shortening scan time of Tc-99m cardiac SPECT perfusion studies. J Nucl Cardiol 2007, 14:555-565.

13. Herzog BA, Buechel RR, Katz R, et al.: Nuclear myocardial perfusion imaging with a cadmium-zinc-telluride detector technique: optimized protocol for scan time reduction. J Nucl Med 2010, 51:46-51.

14. Buechel RR, Herzog BA, Husmann L, et al.: Ultrafast nuclear myocardial perfusion imaging on a new gamma camera with semiconductor detector technique: first clinical validation. Eur J Nucl Med Mol Imaging 2009, In press.

15. • Esteves FP, Raggi P, Folks RD, et al.: Novel solid-state-detector dedicated cardiac camera for fast myocardial perfusion imaging: multicenter comparison with standard dual detector cameras. J Nucl Cardiol 2009, 16:927-934. This is the first clinical US study of the new multi-pinhole detector system.

16. Sharir T, Ben-Haim S, Merzon K, et al.: High-speed myocardial perfusion imaging: initial clinical comparison with conventional dual detector anger camera imaging. JACC Cardiovasc Imaging 2008, 1:156-163.

17. • Berman DS, Kang X, Tamarappoo B, et al.: Stress thallium-201/rest technetium-99m sequential dual isotope high-speed myocardial perfusion imaging. JACC Cardiovasc Imaging 2009, 2:273-282. This is a novel Tl/Tc protocol for dose-efficient fast stress and rest MPS scanning.

18. Nishina H, Slomka PJ, Abidov A, et al.: Combined supine and prone quantitative myocardial perfusion SPECT: method development and clinical validation in patients with no known coronary artery disease. J Nucl Med 2006, 47:51-58.

19. •- Sharir T, Slomka P, Hayes S, et al.: Multicenter trial of high-speed vs. conventional SPECT imaging: quantitative results of myocardial perfusion and left ventricular function. J Am Coll Cardiol 2010, In press. This article includes a comparison of fully automated perfusion quantification for conventional and fast SPECT.

20. Slomka PJ, Nishina H, Berman DS, et al.: Automated quantification of myocardial perfusion SPECT using simplified normal limits. J Nucl Cardiol 2005, 12:66-77.

21. Germano G, Kiat H, Kavanagh PB, et al.: Automatic quantification of ejection fraction from gated myocardial perfusion SPECT. J Nucl Med 1995, 36:2138-2147.

22. Maddahi J, Mendez R, Mahmarian JJ, et al.: Prospective multicenter evaluation of rapid, gated SPECT myocardial perfusion upright imaging. J Nucl Cardiol 2009, 16:351-357.
23. DePuey EG, Bommireddipalli S, Clark J, et al.: Wide beam reconstruction "quarter-time" gated myocardial perfusion SPECT functional imaging: a comparison to "full-time" ordered subset expectation maximum. J Nucl Cardiol 2009, 16:736-752.

24. Cahill JM, Ficaro EP, Corbett JR: 4.11: Value of same-session coronary artery calcium (CAC) scoring in the setting of stress myocardial perfusion imaging (MPI) using CT-based attenuation correction (CTAC). J Nucl Cardiol 2008, 15:12.

25. Koss J, Kirch D, Steele P: Fast acquisition of myocardial perfusion and LV contractile indices with multi-pinhole SPECT [abstract]. J Nucl Med 2009, 50(Suppl 2):1144.

26. Heller GV, Bateman TM, Johnson LL, et al.: Clinical value of attenuation correction in stress-only Tc-99m sestamibi SPECT imaging. J Nucl Cardiol 2004, 11:273-281.

27. Iskandrian AE: Stress-only myocardial perfusion imaging: a new paradigm. J Am Coll Cardiol 2009, In press.

28. Venero CV, Heller GV, Bateman TM, et al.: A multicenter evaluation of a new post-processing method with depthdependent collimator resolution applied to full-time and halftime acquisitions without and with simultaneously acquired attenuation correction. J Nucl Cardiol 2009, 16:714-725.

29. Ali I, Ruddy TD, Almgrahi A, et al.: Half-time SPECT myocardial perfusion imaging with attenuation correction. J Nucl Med 2009, 50:554.

30. • Bateman TM, Heller GV, McGhie AI, et al.: Multicenter investigation comparing a highly efficient half-time stress-only attenuation correction approach against standard rest-stress Tc-99m SPECT imaging. J Nucl Cardiol 2009, 16:726-735. This article demonstrates the feasibility of stress-only fast MPS protocols.

31. Gambhir SS, Berman DS, Ziffer J, et al.: A novel high-sensitivity rapid-acquisition single-photon cardiac imaging camera. J Nucl Med 2009, 50:635

32. Kacperski K, Erlandsson $\mathrm{K}$, Ben-Haim S, et al.: Iterative deconvolution of simultaneous dual radionuclide projections for CdZnTe based cardiac SPECT. IEEE Nuclear Science Symposium Conference Record; 2008:5260-5263.

33. Kiat H, Germano G, Friedman J, et al.: Comparative feasibility of separate or simultaneous rest thallium-201/stress technetium-99msestamibi dual-isotope myocardial perfusion SPECT. J Nucl Med 1994, 35:542.

34. Steele PP, Kirch DL, Koss JE: Comparison of simultaneous dual-isotope multipinhole SPECT with rotational SPECT in a group of patients with coronary artery disease. J Nucl Med 2008, 49:1080

35. Ben-Haim S, Kacperski K, Hain S, et al.: Simultaneous dualradionuclide myocardial perfusion imaging with a solid-state dedicated cardiac camera. Eur J Nucl Med Mol Imaging 2010, In press.

36. Beller GA: Will cardiac positron emission tomography ultimately replace SPECT for myocardial perfusion imaging? J Nucl Cardiol 2009, 16:841-843.

37. Funk T, Kirch DL, Koss JE, et al.: A novel approach to multipinhole SPECT for myocardial perfusion imaging. J Nucl Med 2006, 47:595-602.

38. Huang Q, Boutchko R, Reutter B, Gullberg G: Dynamic imaging with a novel dedicated cardiac SPECT system. J Nucl Med 2009, 50(Suppl 2):525P.

39. Breault C, Roth N, Slomka P, et al.: Quantification of coronary vasodilator reserve using dynamic SPECT imaging: a feasibility study [abstract]. J Nucl Cardiol 2010, (in press).

40. Slomka P, Kavanagh PB, Javadi M, et al.: Evaluation of a new automatic algorithm for quantification of ECG-gated $82 \mathrm{Rb}$ cardiac PET. J Nucl Med 2009, 50(Suppl 2):217P.

41. Slomka PJ, Berman DS, Germano G: Applications and software techniques for integrated cardiac multimodality imaging. Exp Rev Cardiovasc Ther 2008, 6:27-41. 
42. Gutstein A, Wolak A, Lee C, et al.: Predicting success of prospective and retrospective gating with dual-source coronary computed tomography angiography: development of selection criteria and initial experience. J Cardiovasc Comput Tomogr 2008, 2:81-90.

43. - Husmann L, Herzog BA, Gaemperli O, et al.: Diagnostic accuracy of computed tomography coronary angiography and evaluation of stress-only single-photon emission computed tomography/computed tomography hybrid imaging: comparison of prospective electrocardiogram-triggering vs. retrospective gating. Eur Heart J 2009, 30:600. Novel protocols are proposed in this article, which combine MPS and CCTA.

44. DePuey EG: New software methods to cope with reduced counting statistics: shorter SPECT acquisitions and many more possibilities. J Nucl Cardiol 2009, 16:335-338.
45. Achenbach S, Marwan M, Ropers D, et al.: Coronary computed tomography angiography with a consistent dose below $1 \mathrm{mSv}$ using prospectively electrocardiogram-triggered high-pitch spiral acquisition. Eur Heart J 2010, 31:340-346.

46. Santana CA, Garcia EV, Faber TL, et al.: Diagnostic performance of fusion of myocardial perfusion imaging (MPI) and computed tomography coronary angiography. J Nucl Cardiol 2009, 16:201211.

47. Slomka PJ, Cheng VY, Dey D, et al.: Quantitative analysis of myocardial perfusion SPECT anatomically guided by coregistered 64-slice coronary CT angiography. J Nucl Med 2009, 50:1621-1630.

48. Zeng GL, Stevens AM: Multidivergent-beam stationary cardiac SPECT. Med Phys 2009, 36:2860-2869.

49. Dey J: Conference record for IEEE NSS-MIC. 2009, In press. 